\title{
GRB-SN CONNECTION IN SAO RAS OBSERVATIONS
}

\author{
A.S. Moskvitin ${ }^{1}$, V.V. Sokolov ${ }^{1}$, V.N. Komarova et al. ${ }^{1}$
}

\begin{abstract}
We present the results of SAO RAS 6-m BTA spectral observations of GRBs and XRFs with the signs of SNe: GRB/XRF 060218 (SN 2006aj) and XRF 080109 (SN 2008D). The properties of these events are similar to those of usual core-collapse SNe without GRBs. Some spectral features in the cases of SN 2006aj and SN 2008D may be interpreted as hydrogen and helium traces. This supports an idea of presence of a relic envelope around the progenitor star and the shock break-out effects observed in X-rays, UV and optics.
\end{abstract}

\section{GRB-SN}

Work on the search of optical candidates of GRBs started in SAO RAS in 1993. The main aim of this program was to find objects associated with old (archive) bursts with small boxes of localization $\left(\sim 1 \operatorname{arcmin}^{2}\right)$. Because of fast decay of the afterglow brightness and the presence of numerous objects in the error areas the first optical identification with the starforming galaxies was made only in 1997. The next year the GRB follow-up team in cooperation with the Spain team started observing GRB afterglows and their host galaxies (Sokolov 2011).

During recent few years an idea about connection between long-duration GRBs and core-collapse SNe (types Ib and Ic) was supported by numerous observations of such events (Hjorth \& Bloom 2011). However, spectroscopically such an association was carried out only for nearby objects. Apparently, in the visible range this can be reliably done for events at redshifts up to $z \sim 0.5$, when the brightness of an SN (especially at maximum) will still dominate in the overall radiation. It would be interesting to compare properties of SNe with and without GRBs. Here the researchers are facing a problem: why some nearby GRBs are not associated with SNe (Della Valle et al. 2006)?

In SAO RAS we study the evolution of SNe associated with GRBs (and the XRFs), and also usual core-collapse SNe and usual long-duration GRBs without such a link.

1 SAO RAS, Russia; e-mail: mosk@sao.ru; sokolov@sao.ru; vkom@sao.ru 

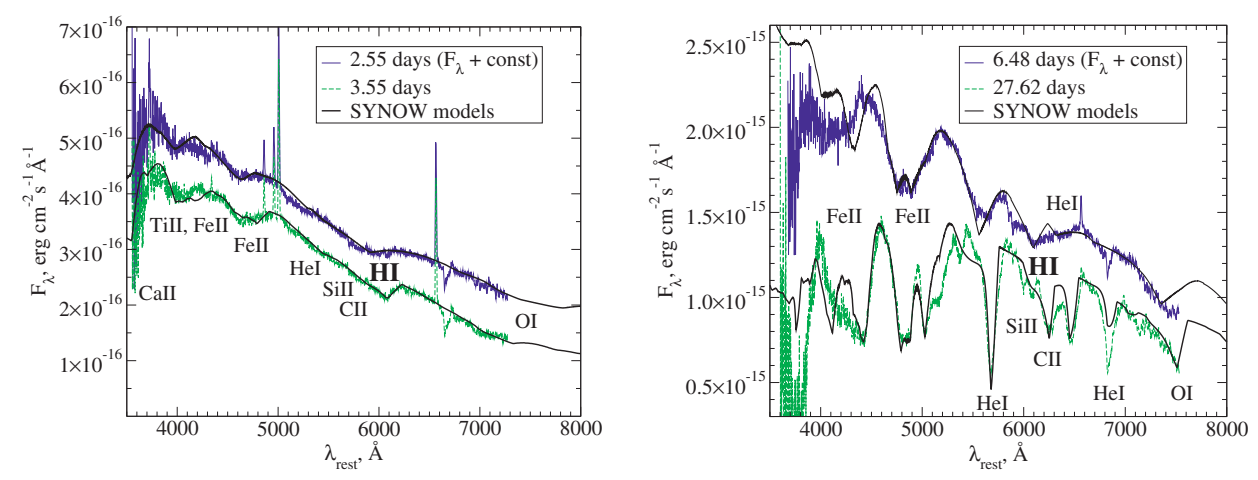

Fig. 1. Left pannel: the spectra of GRB/XRF 060218 (SN 2006aj) obtained with the BTA since $2.55\left(V_{p h}=V_{H I}=V_{H e I}=33000 \mathrm{~km} / \mathrm{s}\right)$ and $3.55\left(V_{p h}=18000 \mathrm{~km} / \mathrm{s} ; V_{H I}=\right.$ $V_{H e I}=24000 \mathrm{~km} / \mathrm{s}$ ) days after the trigger. Right pannel: the spectra of XRF 080109 (SN 2008D) obtained with the BTA since $6.48\left(V_{p h}=V_{H e I}=17000 \mathrm{~km} / \mathrm{s} ; V_{H I}=\right.$ $23000 \mathrm{~km} / \mathrm{s})$ and $27.62\left(V_{p h}=8500 \mathrm{~km} / \mathrm{s} ; V_{H e I}=10500 \mathrm{~km} / \mathrm{s} ; V_{H I}=15000 \mathrm{~km} / \mathrm{s}\right)$ days after the trigger.

International photometrical and spectral monitoring of GRBs and SNe (in collaboration with India, USA, Turkey, Spain, etc.) is ongoing in SAO for many years. Follow-up of early phases of SNe and GRBs, explosion asymmetry, wide luminocity range, hosts, and very distant objects are the most interesting tasks for this programs (Moskvitin et al. 2010b). In the nearest future we plan to make polarimetrical observations too with the BTA/MANIA.

\section{GRB 060218/SN 2006aj and XRF 080109/SN 2008D}

One of the most interesting object was the burst GRB/XRF $060218(z=0.1683)$ with strong X-ray and UV components (dominating in the first hours) and supernova signs (SN 2006aj). These effects can be explained by interaction between SN shock and a stellar-wind envelope around a massive progenitor star (the "shock breakout" which is also visible at early stages of some $\mathrm{SNe}$ ).

The object was observed with the BTA 2.55 and 3.55 days after the trigger. The spectra were modelled in the Sobolev approximation with the SYNOW code (Branch et al. 2003; Elmhamdi et al. 2006). The detected detail near $6200 \AA$ were interpreted as hydrogen line with high velocities: $33000 \mathrm{~km} / \mathrm{s}$ for the first epoch, and $24000 \mathrm{~km} / \mathrm{s}$ for the second one (left pannel of Fig. 1, and Sonbaş et al. 2008). This may be a sign of stellar-wind envelope around a massive progenitor star.

Another significant event observed at SAO RAS with the BTA was the X-ray flash XRF 080109 associated with SN 2008D. This object was also observed spectroscopically with the BTA. The spectra were modelled with the help of SYNOW code to interpret broad features, especially near $6200 \AA$, and to measure expansion velocities. It was shown that Si II can not fit this absorption because of limitation 


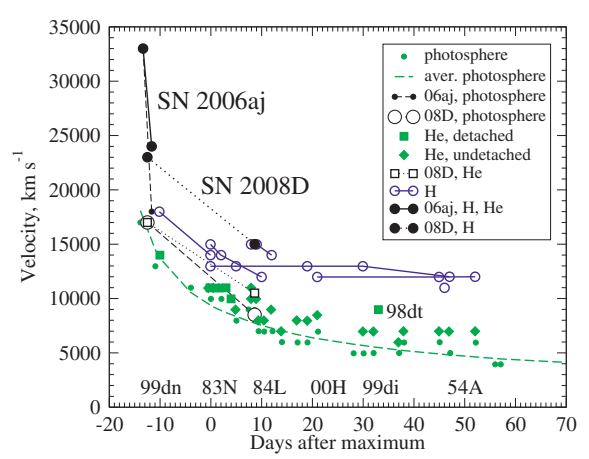

Fig. 2. Comparison of properties of ususal Ib SNe (Branch et al. 2002), SN 2006aj and SN 2008D: expansion velocities of pseudo-photosphere, Helium and Hydrogen.

by the heavier elements such as Fe II. However C II remains a candidate for interpretation of this line (right pannel of Fig. 1, and Moskvitin et al. 2010a).

The assumption about the presence of envelopes around progenitor stars is confirmed by agreement between velocities of lines interpreted as hydrogen and helium, and the empiric power-law velocity drop with time for envelopes of classic core-collapse supernovae (Fig. 2, and Branch et al. 2002). Detection of a $\mathrm{P}$ Cyg profile of the $\mathrm{H} \beta$ line in the spectra of optical afterglows of GRBs can be a conclusive argument in favor of this hypothesis (Moskvitin et al. 2010a).

The authors are grateful to T.N. Sokolova and A.A. Volnova. This work was supported by the RFBR grant 11-02-12696-IND-a; the program No. 17 "Active processes in galactic and extragalactic objects" of the Department of Physical Sciences of the Russian Academy of Sciences; and grants No. 14.B37.21.0251 and No. 14.A18.21.1179 from FTP of the RF Ministry of Education and Science.

\section{References}

Branch, D., Benetti, S., Kasen, D., et al., 2002, ApJ, 566, 1005

Branch, D., Baron, E.A., \& Jeffery, D. J., 2003, chapter in "Supernovae and Gamma-Ray Bursters", ed. Kurt W. Weiler, Lect. Notes Phys. 598, 47 [astro-ph/0111573]

Della Valle, M., Chincarini, G., Panagia, N., et al., 2006, Nature, 444, 1050

Elmhamdi, A., Danziger, I.J., Branch, D., et al., 2006, A\&A, 450, 305

Hjorth, J., \& Bloom, J.S., 2011, Chapter 9 in "Gamma-Ray Bursts", Cambridge Astrophysics Series 51, ed. C. Kouveliotou, R.A.M.J. Wijers \& S. Woosley (Cambridge University Press), p. 169 [arXiv:1104.2274]

Moskvitin, A.S., Sonbaş, E., Sokolov, V.V., et al., 2010, Astrophys. Bull., 65, 132 [arXiv: 1004.2633]

Moskvitin, A.S., Fatkhullin, T.A., Sokolov, V.V., et al., 2010, Astrophys. Bull., 65, 230 [arXiv: 1008.0773]

Sokolov, V.V., 2011, submitted to BASI [arXiv:1111.1406]

Sonbasş, E., Moskvitin, A.S., Fatkhullin, T.A., et al., 2008, Astrophys. Bull., 63, 228 [arXiv:0805.2657] 
